\title{
Shaping Bacterial Symbiosis With Legumes by Experimental Evolution
}

\author{
Marta Marchetti, ${ }^{1,2}$ Alain Jauneau, ${ }^{3,4}$ Delphine Capela, ${ }^{1,2}$ Philippe Remigi, ${ }^{1,2}$ Carine Gris, ${ }^{1,2}$ \\ Jacques Batut, ${ }^{1,2}$ and Catherine Masson-Boivin ${ }^{1,2}$ \\ ${ }^{1}$ INRA, and ${ }^{2}$ CNRS, Laboratoire des Interactions Plantes-Microorganismes, UMR2594, F-31326 Castanet-Tolosan, France; \\ ${ }^{3} \mathrm{CNRS}$, and ${ }^{4}$ UPS, Fédération de Recherches Agrobiosciences, Interactions, Biodiversity, Plateforme d'Imagerie TRI, \\ F-31320 Castanet-Tolosan, France
}

Submitted 21 March 2014. Accepted 30 April 2014.

\begin{abstract}
Nitrogen-fixing symbionts of legumes have appeared after the emergence of legumes on earth, approximately 70 to 130 million years ago. Since then, symbiotic proficiency has spread to distant genera of $\alpha$ - and $\beta$-proteobacteria, via horizontal transfer of essential symbiotic genes and subsequent recipient genome remodeling under plant selection pressure. To tentatively replay rhizobium evolution in laboratory conditions, we previously transferred the symbiotic plasmid of the Mimosa symbiont Cupriavidus taiwanensis in the plant pathogen Ralstonia solanacearum, and selected spontaneous nodulating variants of the chimeric Ralstonia sp. using Mimosa pudica as a trap. Here, we pursued the evolution experiment by submitting two of the rhizobial drafts to serial ex planta-in planta (M. pudica) passages that may mimic alternating of saprophytic and symbiotic lives of rhizobia. Phenotyping 16 cycle-evolved clones showed strong and parallel evolution of several symbiotic traits (i.e., nodulation competitiveness, intracellular infection, and bacteroid persistence). Simultaneously, plant defense reactions decreased within nodules, suggesting that the expression of symbiotic competence requires the capacity to limit plant immunity. Nitrogen fixation was not acquired in the frame of this evolutionarily short experiment, likely due to the still poor persistence of final clones within nodules compared with the reference rhizobium $C$. taiwanensis. Our results highlight the potential of experimental evolution in improving symbiotic proficiency and for the elucidation of relationship between symbiotic capacities and elicitation of immune responses.
\end{abstract}

Legumes and bacteria collectively known as rhizobia have co-evolved a mutualistic symbiosis of major ecological importance that contributes approximately $25 \%$ of global nitrogen cycling (Masson-Boivin et al. 2009). Rhizobia are primarily soil bacteria that are able, when they encounter a compatible host, to induce the organogenesis of root nodules that they colonize intracellularly. Bacteria internalized in nodule cells-

Current address for P. Remigi: New Zealand Institute for Advanced Study, Massey University, Auckland, New Zealand.

Corresponding author: C. Masson-Boivin;

E-mail: catherine.masson@toulouse.inra.fr

* The $e$-Xtra logo stands for "electronic extra" and indicates that four supplementary figures and one supplementary table are published online.

(C) 2014 The American Phytopathological Society called bacteroids-persist and fix nitrogen, resulting in a chronic intracellular infection and symbiosis (Fones and Preston 2012; Gibson et al. 2008; Ivanov et al. 2010). When nodules senesce, bacteria are released to the soil, in which they compete with other bacteria for survival and colonization of a new host (Timmers et al. 2000).

Rhizobia belong to various genera of $\alpha$ - and $\beta$-proteobacteria (Gyaneshwar et al. 2011; Moulin et al. 2001; Sy et al. 2001), indicating that a large diversity of bacteria has developed the ability to enter into a nitrogen-fixing symbiosis with legumes during evolutionary times (Masson-Boivin et al. 2009). The spread of symbiotic traits to bacteria adapted to various environments and host plants has likely favored the success of this symbiosis, which involves more than $70 \%$ of the legumes (Sprent 2001, 2007). Key nodulation and nitrogen fixation genes required for legume symbiosis have been acquired by horizontal transfer, as a first step in converting soil bacteria to mutualistic symbionts (Galibert et al. 2001; Hirsch et al. 2001; Ramsay et al. 2009; Rogel et al. 2001; Sullivan and Ronson 1998). Many other genes, including species-specific genes, have been recruited in the recipient genome for symbiosis (Amadou et al. 2008; Tian et al. 2012). In many instances (i.e., when the donor and receiver of key nod and nif genes were phylogenetically distant), full expression of the symbiotic potential may have relied on the reprogramming of the recipient genome (Marchetti et al. 2010; Masson-Boivin et al. 2009). Alternation of saprophytic and symbiotic lives may have shaped rhizobia along with evolution, likely by allowing the legume host to select the most beneficial variants at each new infection. However, very few experimental data support this scheme thus far.

Experimental evolution, which involves the propagation of bacterial populations over hundreds of generations in a controlled environment (Barrick and Lenski 2013; Buckling et al. 2009), is a powerful tool to tentatively replay rhizobium evolution in real time and analyze the evolutionary forces that have driven natural selection of legume symbionts. We previously initiated the conversion of the plant root bacterial pathogen Ralstonia solanacearum into a legume symbiont by introducing the symbiotic plasmid of the rhizobium Cupriavidus taiwanensis into $R$. solanacearum strain GMI1000 and by using the C. taiwanensis host, Mimosa pudica, as selection pressure to trap nodulating derivatives (Marchetti et al. 2010). Among the three spontaneous variants that had gained the ability to nodulate $M$. pudica (following one cycle of massive inoculation), CBM212 and CBM349 were also able to intracellularly infect nodules, although some variability was observed depending on the $M . p u$ dica geographical origin. Infection was, however, rudimentary compared with the $M$. pudica symbiont $C$. taiwanensis. Fewer 
bacteria were routinely isolated per nodule, bacteroids did not fix nitrogen or persist, and bacteria were found in intercellular spaces of the nodule (Marchetti et al. 2010).

Here, we pursued the evolution experiment by progressively adapting Ralstonia CBM212 and CBM349 to nodule tissues using serial ex planta-in planta cycles. We showed, using comparative phenotyping of ancestral and evolved clones, that serial ex planta-in planta passages greatly improved nodulation and intracellular infection of the rhizobial drafts CBM212 and CBM349 and simultaneously reduced their capacity to induce plant defense reactions in nodules. Our study shows that rhizobia can be shaped by experimental evolution and provides experimental evidence that emerging rhizobia evolved to reduce stimulation of host defenses.
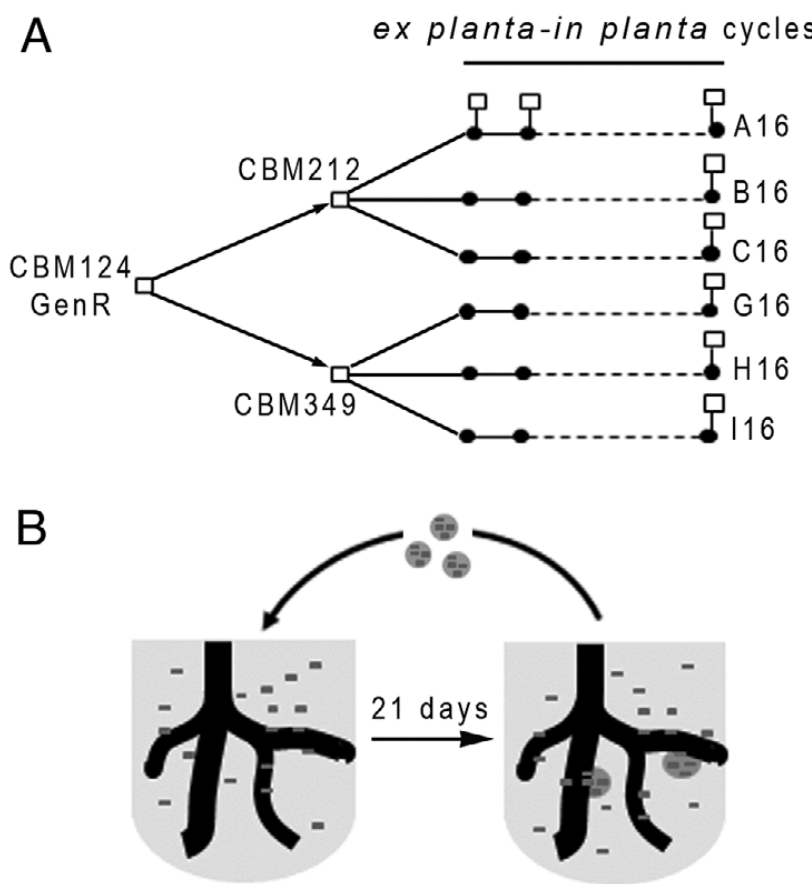

Fig. 1. Evolution of symbiotically improved Ralstonia spp. A, CBM212 and CBM349, two spontaneous Mimosa pudica-nodulating derivatives of the chimeric Ralstonia CBM124GenR, were evolved using serial ex planta-in planta cycles. Six independent lineages have been derived from CBM212 (lineages A, B, and C) and CBM349 (lineages G, H, and I). B, In each cycle, bacteria were inoculated to 10 tubes containing $M$. pudica plantlets grown in favorable N-starvation conditions. At 21 days after inoculation, a sample of the bacterial population recovered from all nodules was inoculated to a new set of plants.

\section{RESULTS}

Sixteen cycles of lab evolution

under Mimosa selection pressure improved nodulation and infection of chimeric Ralstonia variants.

$M$. pudica is the specific host of $C$. taiwanensis (Chen et al. 2003; Elliott et al. 2009), the donor of symbiotic plasmid in our experimental system. To improve their symbiotic properties, Ralstonia CBM212 and CBM349 were progressively adapted to $M$. pudica nodule tissues by serial ex planta-in planta cycles (Fig. 1). Briefly, the ancestral strains were inoculated to $M$. pudica plantlets grown in nitrogen-free conditions and, every 21 days, samples of the bacterial population recovered from all nodules were inoculated to a new set of plants. Six independent lineages of 16 cycles have been derived from CBM212 (lineages A, B, and C) and CBM349 (lineages G, H, and I) (Fig. 1). In each final nodule population, one representative clone was isolated and kept at $-80^{\circ} \mathrm{C}$ to serve as a "living fossil record" for further analysis and comparison with its ancestor, either CBM212 or CBM349.

To evaluate global changes in bacterial symbiotic ability after 16 cycles, each pair of ancestral/final clones was co-inoculated to $M$. pudica at a 1:1 ratio and the relative in planta fitness of the clones was estimated by counting the respective number of ancestors and final clones recovered from the nodules. For each lineage, final clones represented more than $95 \%$ of the nodule population (Table 1).

Increase in in planta fitness was attributable to improvement of both nodulation and infection capacities. Approximately one $\log$ more bacteria were isolated per nodule for final clones B16, C16, G16, H16, and I16 than for their respective ancestors (Fig. 2A), indicating that infection capacity in a broad sense was greatly improved along the experiment in the corresponding lineages. Following co-inoculation of pairs of ancestral/final clones on $M$. pudica at a 1:1 ratio, nodules formed by final clones represented 84 to $98 \%$ of total nodules in all cases (Table 1), showing that nodulation competitiveness (i.e., ability to successfully compete with ancestors for nodulation of host plants) has been strikingly improved after 16 cycles. Yet the final clones were not symbiotically equivalent. Co-inoculation experiments revealed that $\mathrm{B} 16$ and $\mathrm{C} 16$ were the fittest clones among the CBM212-derived lineages, while G16 and I16 were the fittest CBM349-derived final clones (Table 1).

However, evolution was far from complete. One of the best final clones, B16, was still approximately 100 times less fit than C. taiwanensis (Table 1). None of the final clones fixed nitrogen as assessed by acetylene reduction assays, and fresh sections of nodules were white, as observed for a nifH mutant of C. taiwanensis (data not shown). Accordingly, nodules

Table 1. Relative in planta fitness and nodulation competitiveness of evolved clones ${ }^{\mathrm{a}}$

\begin{tabular}{|c|c|c|c|c|c|c|c|c|c|c|}
\hline \multirow[b]{2}{*}{$\operatorname{Mix}(1 / 2 / 3)$} & \multirow[b]{2}{*}{ Ratio } & \multicolumn{4}{|c|}{ Relative fitness } & \multicolumn{5}{|c|}{ Nodulation competitiveness } \\
\hline & & $1(\%)$ & $2(\%)$ & $3(\%)$ & $n$ & $1(\%)$ & $2(\%)$ & $3(\%)$ & Empty (\%) & $n$ \\
\hline A16/CBM212 & $1: 1$ & $98.5 \pm 0.9$ & $1.5 \pm 0.9$ & $\cdots$ & 3 & $84.3 \pm 8.9$ & $5.3 \pm 0.2$ & $\ldots$ & $10.4 \pm 9.1$ & 2 \\
\hline B16/CBM212 & $1: 1$ & $99.9 \pm 0.1$ & $0.1 \pm 0.1$ & $\ldots$ & 3 & $96.5 \pm 1$ & $0.7 \pm 1$ & $\ldots$ & $2.8 \pm 2$ & 2 \\
\hline C16/CBM212 & $1: 1$ & $97.1 \pm 2.7$ & $2.9 \pm 2.7$ & $\ldots$ & 2 & $93.9 \pm 2.2$ & $3.8 \pm 2.1$ & $\ldots$ & $2.2 \pm 0.05$ & 2 \\
\hline G16/CBM349 & $1: 1$ & $99.8 \pm 0.2$ & $0.2 \pm 0.2$ & $\ldots$ & 4 & $97.5 \pm 0.1$ & $1.2 \pm 0.8$ & $\ldots$ & $1.3 \pm 0.7$ & 2 \\
\hline H16/CBM349 & $1: 1$ & $95.8 \pm 2.5$ & $4.2 \pm 2.5$ & $\ldots$ & 2 & $92 \pm 7.1$ & $6.3 \pm 5.2$ & $\ldots$ & $1.7 \pm 1.9$ & 2 \\
\hline I16/CBM349 & $1: 1$ & $99.9 \pm 0.1$ & $0.06 \pm 0.1$ & $\ldots$ & 3 & $98.3 \pm 1.6$ & $0.6 \pm 0.3$ & $\ldots$ & $1.1 \pm 1.4$ & 2 \\
\hline A16/B16/C16 & $1: 1: 1$ & $1.8 \pm 0.2$ & $49.3 \pm 19.7$ & $48.9 \pm 19.5$ & 3 & $3.2 \pm 0.8$ & $42.6 \pm 17.6$ & $46 \pm 17.1$ & $8.3 \pm 1.8$ & 3 \\
\hline G16/H16/I16 & $1: 1: 1$ & $52.7 \pm 22.6$ & $0.9 \pm 1.1$ & $46.3 \pm 22.4$ & 3 & $59.4 \pm 20.6$ & $2.7 \pm 3.3$ & $31.9 \pm 17.6$ & $6 \pm 2.3$ & 3 \\
\hline LMG19424/B16 & $1: 1$ & $94.9 \pm 7.9$ & $5.1 \pm 7.9$ & $\ldots$ & 4 & $\ldots$ & $\ldots$ & $\ldots$ & $\ldots$ & .. \\
\hline
\end{tabular}

\footnotetext{
${ }^{\text {a }}$ Fitness of a clone is presented as the normalized in-planta fitness of this clone divided by the sum of the normalized in-planta fitnesses of all clones. The normalized in-planta fitness of a clone corresponds to the percentage of bacterial cells in the nodule population divided by the percentage of bacterial cells in the inoculum. Values are averages \pm standard deviation. Nodules were collected at 21 days postinoculation from six plants for relative fitness and from 20 plants for nodulation competitiveness; $n=$ number of independent experiments; Empty = empty nodules; LMG19424 = C. taiwanensis wild-type strain; other clones $=$ chimeric Ralstonia spp.
} 
formed by final clones exhibited a lower level of leghemoglobins, which are plant proteins involved in nodule development and functioning, than observed with the wild-type $C$. taiwanensis strain or an isogenic nifH mutant (Fig. 3). Yet the leghe-
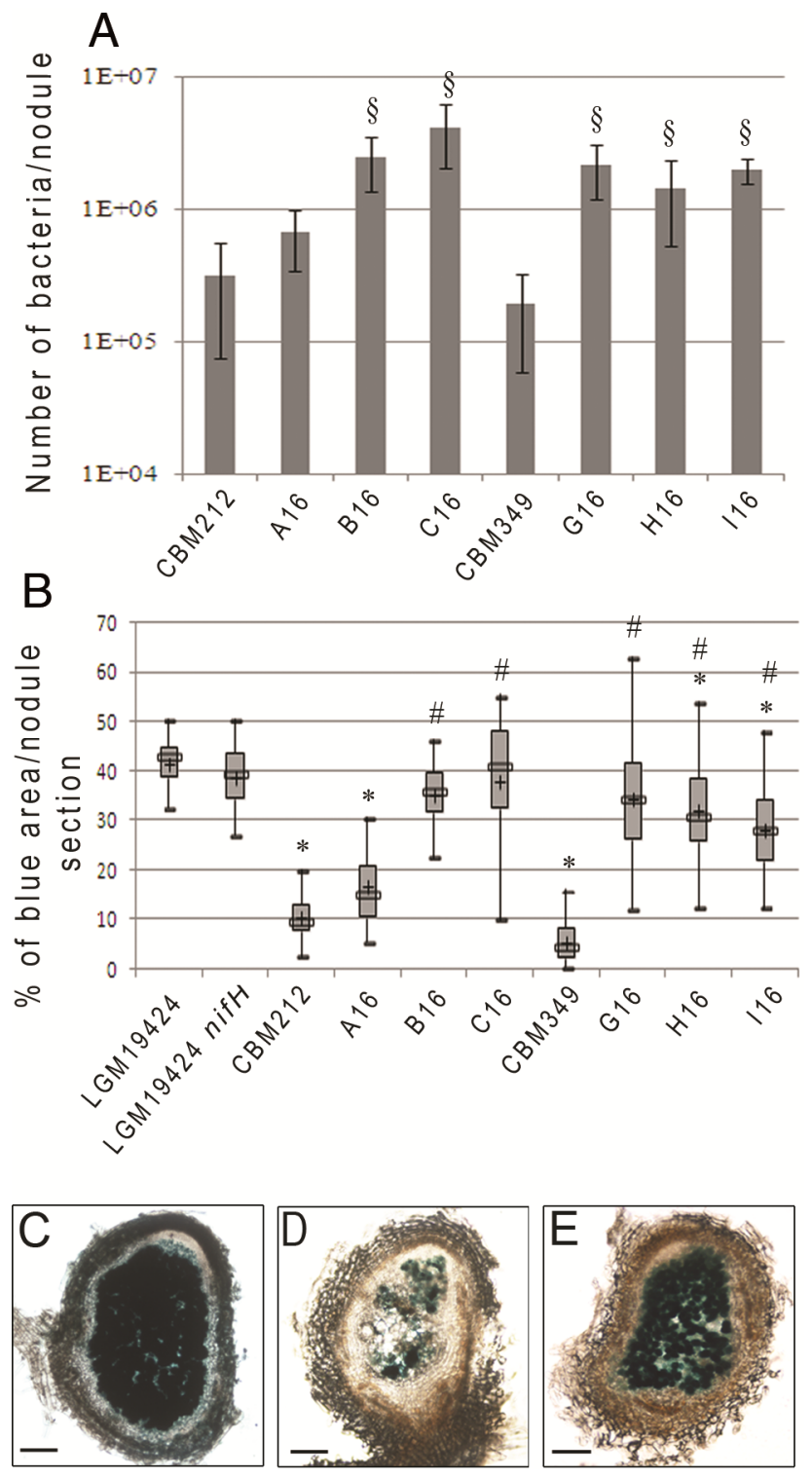

Fig. 2. Infection phenotyping of evolved clones. A, In planta fitness of evolved clones. Values are averages \pm standard deviation of the number of bacteria recovered per nodule from three independent experiments. In each experiment, nodules from 6 plants were harvested 21 days after inoculation (dpi). Symbol $\S$ indicates significant differences with the relative ancestor $(P<0.05$; Kruskal-Wallis test). B through E, Sections of 21-dpi Mimosa pudica nodules induced by the ancestral and evolved strains carrying a constitutively expressed nodB-gusA fusion and by Cupriavidus taiwanensis LMG19424 or the nifH mutant CBM2432 carrying a nodB-lacZ fusion (CBM132 and CBM2543, respectively) were used for infection analysis, as shown for C, C. taiwanensis; D, CBM212; and E, B16. B, For infection quantification, the blue areas of nodule sections were measured using the Image Pro software and divided by the total nodule surface. Box plots represent the distribution of the percentages of infection obtained from 25 to 39 nodules analyzed per strain in two to three independent experiments. Scale bar $=100 \mu \mathrm{m}$; *indicates statistically significant difference from the reference rhizobial strain $C$. taiwanensis and \# represents statistically significant difference from the respective ancestral strain $(P<$ 0.05; Kruskal-Wallis test). The central rectangle spans the first quartile to the third quartile (i.e., the interquartile range [IQR]), the segment inside the rectangle shows the median and + indicates the mean; whiskers above and below the box show either the locations of the minimum and maximum in the absence of suspected outlying data. moglobin level was slightly higher than in nodules induced by the CBM212 and CBM349 ancestors (Fig. 3). In addition, nitrogen fixation genes were expressed as shown by visualizing the expression of the nitrogenize gene nifH in $M$. pudica nodules formed by B16 (Supplementary Fig. S1), indicating that sufficient micro-toxic conditions were reached within nodules (Dixon and Kahn 2004). Lack of nitrogen fixation was unlikely to be due or solely due to mutations in the plasmid nif/fix genes because the replacement of prate by the original one from $C$. taiwanensis in B16 or C16 did not lead to nitrogen fixation capacity (Supplementary Fig. S2).

\section{Final clones have increased nodule cell infection and bacteria persistence capacities.}

Gain in infection could result from increase in the number of endosymbiosis bacteria, improvement of their persistence, or both. Changes in infection were evaluated by measuring the surface of the infected zone on sections of nodules collected 21 days postinoculation (dpi). Measures were performed by probing expression of strains bearing a constitutively expressed goose or lacs fusion on one longitudinal section per nodule. The nodule infection area of the B and C (ancestor CBM212) and $\mathrm{G}, \mathrm{H}$, and I (ancestor CBM349) final clones was significantly increased, up to sevenfold, compared with their relative ancestors (Fig. 2B). Notably, increase in infection was mainly due to an increase in intracellular infection, as appreciated by microscopy observations (Fig. $2 \mathrm{C}$ to E).

Similar results were obtained when measuring the lightbrown infected zone of nodule sections by the hue, saturation, and intensity (HSI) method that allows color distinction (see Materials and Methods). However, the difference between final and ancestral clones was lower than with X-glum staining (Supplementary Fig. S3). Differences could come from X-glum staining biases such as staining defect of dead or degraded bacteria and staining excess in the case of high bacterial density. In both cases, infection capacity of B16, C16, and G16 was not statistically different from the reference strain $C$. taiwanensis (Fig. 2B).

Changes in bacteria persistence were investigated in lineages $\mathrm{B}$ and $\mathrm{G}$ by comparing the viability of final and ancestral clones within $M$. pudica nodules using a live/dead staining procedure (Vista et al. 1998) (Fig. 4). Quantification analysis showed that, in 7-day-old nodules, the percentage of live bacteria was significantly higher for B16 and G16 than for their relative ancestors, indicating that these final clones have improved bacteria persistence (Fig. 4I). This difference disappeared in 14-day-old nodules. At both times, however, B16 and G16 were less persistent than the wild-type reference strain C. taiwanensis or even its nifH mutant (Fig. 4J), indicating that their premature senescence within nodules was not just a consequence of inability to fix nitrogen.

Altogether, these results showed that final clones have gained in intracellular infection capacity, including both infections per se and persistence, yet likely still insufficiently to sustain an effective symbiosis.

Final clones induce fewer plant defense reactions in nodules.

In nodules formed by CBM212 and CBM349, a dark-brown zone, never observed in nodules formed by $C$. taiwanensis or its nifH mutant, was often observed inside the nodule and close to the infected zone (Fig. 5A to C, F, and G) (Marchetti et al. 2010). Ultra structural analysis by electron microscopy showed that this brown zone consisted of a hole framed by dead nodule cells (Fig. 5C and D) and was associated with cell wall thickening (Fig. 5E). Necrotic zones were also associated with reactive oxygen species (ROS) production, which is an indicator of plant defenses (Fones and Preston 2012; Lamb 
and Dixon 1997), as visualized using $2^{\prime}, 7^{\prime}$-dichlorodihydrofluorescein diacetate (H2DCFDA) as a probe (Fig. 5I to K). Thus, ancestors induced plant defense reactions when they colonized the formed nodules.

To compare the capacity of ancestral and final clones to induce nodule necrosis, strains were inoculated onto $M$. pudica seedlings and nodule sections were analyzed 21 dpi using two different methods: a qualitative method based on expert identification of the presence of necrotic areas by visual observation, and a quantitative method based on measurement of necrotic areas by automated image analysis. Both analyses showed that the number and surface of the necrotic zones significantly decreased in all evolved clones compared with their respective ancestors (Fig. 5L and M). It is noteworthy that three clones (B16, C16, and G16) were found to induce very few nodule defense reactions. However, they were different from the reference strain C. taiwanensis LMG19424 and its nifH mutant, as assessed by the expert qualitative method.

To evaluate whether final clones had altered capacity to induce plant basal immunity in $R$. solanacearum-compatible hosts, we used a protection assay (Feng et al. 2012) measuring the ability of avirulent bacteria to protect susceptible Arabidopsis thaliana plants from a subsequent attack by a virulent wild-type strain. Preinoculation with the nodulating ancestor CBM212 protected the plant like an avirulent $h r p G$ mutant, whereas the final evolved clones B16 and $\mathrm{C} 16$ did not protect more than water (Supplementary Fig. S4). Interestingly, A16, which is the less infectious evolved clone (Fig. 2A and B), behaved like the CBM212 ancestor. This suggests a correlation between the improvement of symbiotic properties and the loss of capacity to induce plant basal immunity.

\section{DISCUSSION}

The plant-associated microbial community, including commensals, symbionts, and pathogens, is crucial for plant health. Plant-microbe association or functional studies revealed that plants are able to structure their microbiome either by directly

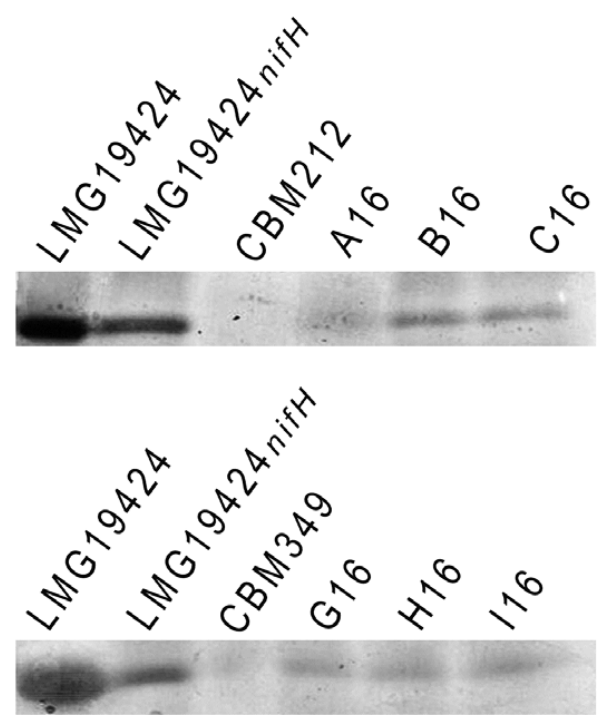

Fig. 3. Leghemoglobin expression. Nodules induced by Cupriavidus taiwanensis wild-type strain LMG19424, C. taiwanensis nifH mutant CBM2432, ancestral clones CBM212 and CBM349, and evolved clones A16-C16 and G16-I16 were used for leghemoglobin expression analysis. Proteins $(20 \mu \mathrm{g})$ from lysates of nodules (21 days after inoculation) were electrophoresed on sodium dodecyl sulfate polyacrylamide gel electrophoresis gels and Western blot detection of leghemoglobin was performed using the anti-legC antibody. modulating the rhizospheric or endophytic compartment microbial composition (Berendsen et al. 2012; Bulgarelli et al. 2012; Lundberg et al. 2012) or by allowing specific in planta multiplication of privileged parasites or symbionts (Oldroyd and Downie 2008). On the microbial side, the capacity of bacteria to adapt to changing environments, due to their high genome plasticity and reproductive rate, may facilitate the evolution of specific interactions.

Here, we show that rounds of ex planta-in planta stages that reasonably mimicked natural alternating of soil and endosymbiotic lives of rhizobia improved symbiotic capacities. Indeed, serial Mimosa-bacteria co-culture cycles allowed rapid (400 generations) and striking simultaneous improvement of several symbiotic traits (i.e., nodulation competitiveness and nodule development, intracellular infection, and bacteroid persistence) of a draft rhizobium exhibiting rudimentary infection capacity. Final clones exhibited differences in the extent of symbiotic improvement, likely attributable to chance events of different random mutations (Lenski and Travisano 1994). Yet parallel symbiotic changes in all lineages indicate that the legume environment is highly selective for the improvement, in addition to activation (Guan et al. 2013; Marchetti et al. 2010), of nodulation and intracellular infection, reinforcing the idea that, following the initial transfer of key symbiotic genes, subsequent genome adaptation under plant selection pressure has been cru-
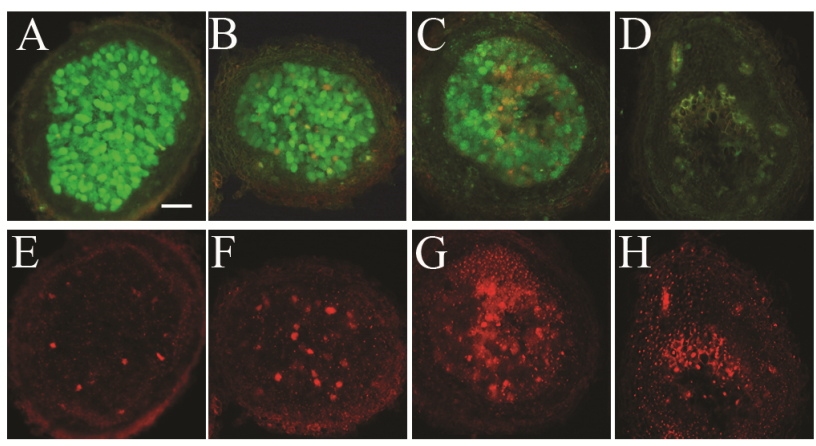

I

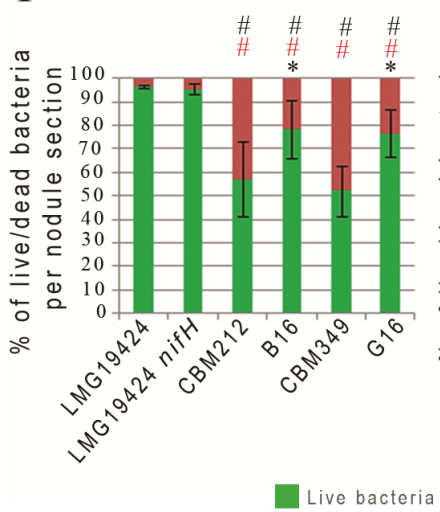

$\mathrm{J}$

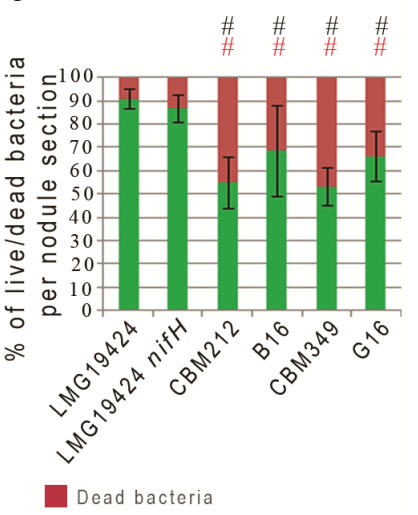

Fig. 4. Persistence of endosymbiotic evolved clones. Live/dead staining of Mimosa pudica nodules formed by $\mathbf{A}$ and $\mathbf{E}$, Cupriavidus taiwanensis LMG19424 and $\mathbf{B}$ and F, its nifH mutant CBM2432; C and G, Ralstonia G16, and D and H, CBM349. A through H, Fresh-sliced nodules harvested at 7 or 14 days post-nodule formation (dpnf) were stained with Syto9 (green signal, live bacteria) (A to D) and PI (red signal, dead bacteria) (E to $\mathrm{H}$ ). I and $\mathbf{J}$, Sections were observed using fluorescent microscopy and used for bacterial viability quantification. The percentage of live/dead bacteria per nodule section was evaluated at I, 7 and J, 14 dpnf. In all, 14 to 30 nodules per strain and per condition were analyzed in two independent experiments. Black \#, red \#, and * indicate significant differences with the $C$. taiwanensis wild-type strain, the $C$. taiwanensis nifH mutant, and their respective ancestor, respectively $(P<0.05$; Student's $t$ test). Scale bar $=100 \mu \mathrm{m}$. 
cial for the evolution and diversification of rhizobia (Capela et al. 2013). Our lab evolution of Ralstonia strains displayed characteristic parallel phenotypic changes of experimental evolution (Le Gac et al. 2013). Yet evolution was extremely rapid compared with other evolution experiments (Barrick et al. 2009), with the final clones being at least 20 times fitter than their respective ancestors in less than 400 generations (Table 1).

In compatible interactions, rhizobia are recognized as alien organisms (Moreau et al. 2011) but do not elicit plant defense reactions while entering root hairs and massively invading nodule cells. It has been shown that both partners have developed strategies to limit and control plant defense reactions
(Mithofer 2002; Saeki 2011; Zamioudis and Pieterse 2012). Here, we experimentally showed that emerging rhizobia evolved to reduce stimulation of host immune responses in response to host selection pressure. Ralstonia ancestors are not pathogens on Mimosa spp. and on other plants thanks to inactivation of the master virulence regulator HrpG (Marchetti et al. 2010). However, they trigger an immune response within nodules which is manifested by the presence of a necrotic zone, a phenomenon that can happen in nature when appropriate partners are absent (Gehlot et al. 2013). The presence and size of nodule necroses strongly diminished in all final clones, which simultaneously exhibited improved symbiotic proficiency.
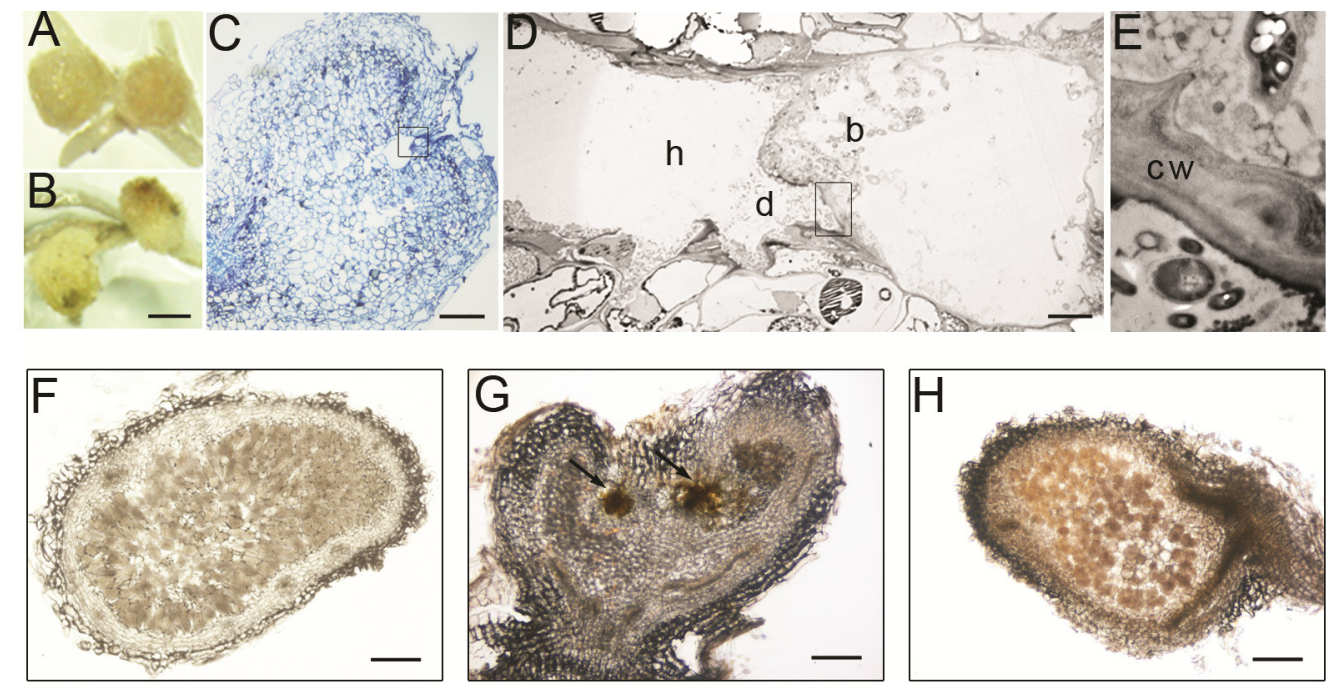

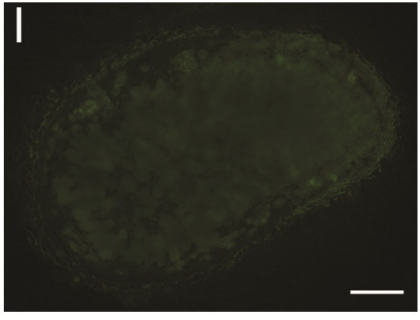

$\mathrm{L}$

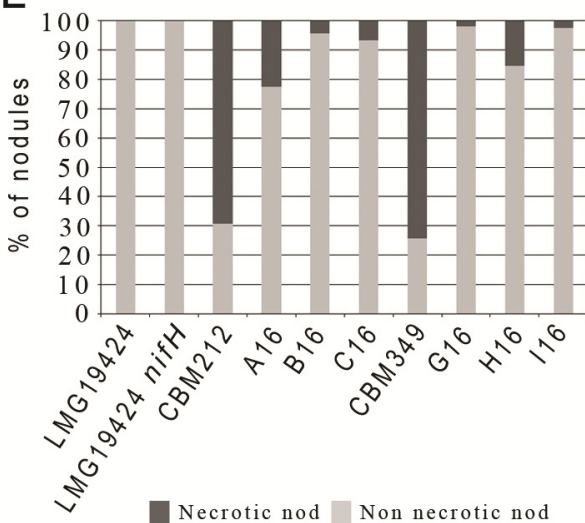

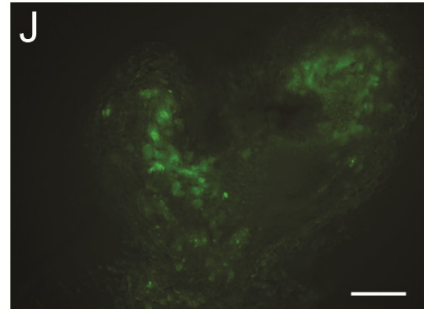

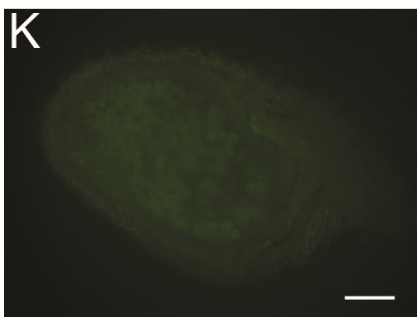

M

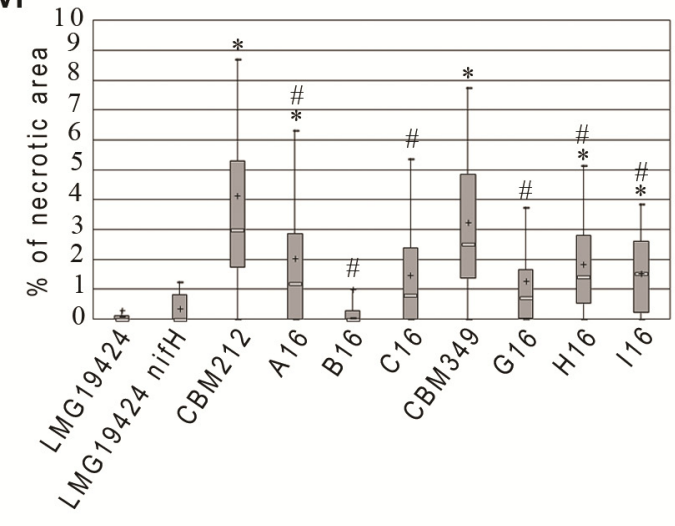

Fig. 5. Nodule necrosis phenotyping of evolved clones. Nodules formed by A, B16 and B, CBM212 on Mimosa pudica. Scale bar = $1 \mathrm{~mm}$. C, Toluidine blue staining of semithin section of nodule formed by CBM212. Scale bar $=100 \mu \mathrm{m}$. D, Magnification of the black square of C. Scale bar $=10 \mu \mathrm{m}$. E, Magnification of the black rectangle in D. Sections of nodules formed by $\mathbf{F}$ and $\mathbf{I}$, Cupriavidus taiwanensis LMG19424; $\mathbf{G}$ and $\mathbf{J}, \mathbf{C B M} 212$; and $\mathbf{H}$ and $\mathbf{K}$, B16 as visualized under bright field microscopy $(\mathrm{F}$ to $\mathrm{H})$ and after reactive oxygen species probing by $2^{\prime}, 7^{\prime}$-dichlorodihydrofluorescein diacetate $(\mathrm{I}$ to $\mathrm{K}$ ); $\mathrm{h}=\mathrm{hole}, \mathrm{d}=$ dead cells, $\mathrm{b}=$ bacteria, $\mathrm{cw}=$ cell wall, and black arrow $=$ dark-brown necrotic zone. Scale bar $=100 \mu \mathrm{m}$. $\mathbf{L}$ and $\mathbf{M}$, Pictures of nodule sections obtained by brightfield microscopy (as in $\mathrm{F}$ to $\mathrm{H}$ ), including the $C$. taiwanensis nifH mutant CBM2432 and all the final evolved clones, were used to determine the presence $(\mathrm{L})$ or the relative surface of the necrotic zone (M). Box plots represent the distribution of the percentages of nodule necrosis per nodule section area obtained from 30 to 52 nodules analyzed per strain in two to three independent experiments; * and \# indicate statistically significant differences with the reference strain $C$. taiwanensis and the respective ancestral strain, respectively $(P<0.05$; Kruskal-Wallis test). The central rectangle spans the first quartile to the third quartile (i.e., the interquartile range [IQR]), the segment inside the rectangle shows the median and + indicates the mean, and whiskers above and below the box show either the locations of the minimum and maximum in the absence of suspected outlying data. 
Rhizobial immune evasion strategies include modification of common conserved structures called microbe-associated molecular patterns (MAMPs) (Felix et al. 1999), which induce a first line of defense against nonadapted invaders (Jones and Dangl 2006), and production of specific extracellular polysaccharides, Nod factors, and type III secretion system (T3SS) effectors, which may actively suppress the immune response (Jones et al. 2007; Zamioudis and Pieterse 2012). In our system, ancestors produce active Nod factors and possess a nonfunctional T3SS. Escaping recognition could occur through evolutionary alteration of MAMPs, a hypothesis supported by the fact that final evolved clones no longer protected susceptible $A$. thaliana plants from a subsequent attack by a virulent wild-type $R$. solanacearum strain. Alternatively, bacteria could have evolved ways to limit plant defenses (i.e., via modulation of extracellular polysaccharides). The fact that, in all lineages, symbiotic improvement is accompanied by reduction in plant defense responses suggests that escaping plant defenses contributes to the expression of the symbiotic traits (i.e., nodulation and infection) acquired by horizontal transfer of nodulation genes. Thus, lab evolution of rhizobia in a legume environment, which exerts a strong selective pressure for unpunished but controlled infection, is a powerful tool to analyze how bacteria evolve to circumvent recognition and defense reactions as well to decipher relationships between symbiosis and plant immunity.

Nitrogen fixation is the ultimate adaptive trait that turns an infective parasitic bacterium into a mutualistic one. Mutualism, which requires that both partners are closely matched for infection, optimum development, nutrient exchange, and nitrogen fixation (Terpolilli et al. 2012; Udvardi and Poole 2013), was not achieved after 16 cycles in our experiment, although nif genes were expressed within nodules. Absence of measurable nitrogen fixation, which requires massive and persistent infection, could be solely due to suboptimal infection capacity. Indeed, final clones do not behave like a nifH mutant of the rhizobium $C$. taiwanensis. They persisted less within nodule cells and induced more nodule necroses, suggesting that bacteria are still undesirable and prematurely killed by the plant. Defects in bacteroid development (although $C$. taiwanensis symbionts of Mimosa spp. are not terminally differentiated) (Marchetti et al. 2011) or metabolism could also be responsible for the lack of nitrogen fixation. Pursuing the evolution experiment should allow further improvement of persistence and, possibly, nitrogen fixation. It has been demonstrated that legumes sanction non-nitrogen-fixing endosymbiotic bacteria (Kiers et al. 2003; Oono et al. 2011), which should favor the emergence of nitrogen-fixing clones. Understanding how to experimentally evolve nitrogen fixation would lead to both fundamental and practical issues regarding the emergence of mutualism in plant-microbe interactions.

\section{MATERIALS AND METHODS}

\section{Bacterial strains, plasmids, and growth conditions.}

Bacterial strains and plasmids used in this work are listed in Supplementary Table S1. $R$. solanacearum strains were grown at $28^{\circ} \mathrm{C}$ on rich $\mathrm{BG}$ medium supplemented with $28 \mathrm{mM}$ glucose (Boucher et al. 1985). Representative clones of nodule bacterial populations were isolated as described (Guan et al. 2013). Antibiotics were used at the following concentrations: spectinomycin at $40 \mu \mathrm{g} / \mathrm{ml}$, trimethoprim at $100 \mu \mathrm{g} / \mathrm{ml}$, tetracycline at $10 \mu \mathrm{g} / \mathrm{ml}$, gentamicin at $10 \mu \mathrm{g} / \mathrm{ml}$, chloramphenicol at $12.5 \mu \mathrm{g} / \mathrm{ml}$, and kanamycin at $50 \mu \mathrm{g} / \mathrm{ml}$.

Generation of symbiotically evolved clones and populations.

The ancestral strains CBM212 and CBM349 grown overnight in BG medium supplemented with trimethoprim were each inoculated to three sets of $30 \mathrm{M}$. pudica plants (3 plants per tube), and $10^{7}$ bacteria per tube were used as inoculum. At 21 dpi, all nodules from each set of 30 plants were pooled, sterilized with $2.6 \%$ sodium hypochlorite for $15 \mathrm{~min}$, and crushed. Then, $10 \%$ of the nodule crush was used to inoculate a new set of 30 plants the same day, except in cycle 7, where plants were inoculated with a 48-h culture of frozen nodule crushes obtained in cycle 6 . At each cycle, serial dilutions of each nodule crush were plated and one clone was randomly selected from the highest dilution and purified. The selected clones, the rest of the nodule crushes, and a 48-h culture of bacteria from an aliquot of nodule crushes were stored at $-80^{\circ} \mathrm{C}$.

\section{Strain construction.}

Spectinomycin- and kanamycin-resistant derivatives of evolved clones were constructed as described (Guan et al. 2013).

To construct the pnodB-gusA and pnifH-gusA genomic fusions, we amplified DNA fragments of $\operatorname{nodB}$ and nifH promoter regions using the couples of primers oCBM1034oCBM1035 and oCBM1697-oCBM1033, respectively, and cloned it into the Bam $\mathrm{HI}$ and $\mathrm{XbaI}$ restriction sites of the integrative plasmid pVO155 (Oke and Long 1999). The resulting plasmids pCBM41 and pCBM96 were introduced into the ancestral and evolved clones of $R$. solanacearum by triparental mating. Transconjugants were selected on BG medium containing trimethoprim and kanamycin antibiotics.

To construct the $C$. taiwanensis nifH mutant (CBM2432), we amplified an internal fragment of the nifH gene using the oCBM21-oCBM22 pair of primers and cloned it into the $B a m \mathrm{HI}$ and $\mathrm{XbaI}$ restriction sites of the integrative plasmid pVO155. The resulting plasmid pCBM151 was introduced into the $C$. taiwanensis CBM832 strain by triparental mating using the pRK600 as helper plasmid. Transconjugants were selected on BG medium containing streptomycin and kanamycin antibiotics. The CBM2432 mutant was verified by polymerase chain reaction (PCR) and tested on $M$. pudica plants to check its inability to fix nitrogen. To construct the $C$. taiwanensis nifH pnodB-LacZ strain (CBM2543), the pCBM01 plasmid (Marchetti et al. 2010) was introduced by triparental conjugation using the pRK600 as helper plasmid and transconjugants were selected by their tetracycline resistance.

Exchange of evolved pRalta (trimethoprim resistant) by a wild-type pRalta in final evolved clones was achieved by conjugation with the donor $C$. taiwanensis CBM61 strain carrying the symbiotic plasmid pRalta::Tn5-B13S (tetracycline resistant) and the helper plasmid RP4-7. Transconjugants resistant to tetracycline and sensitive to trimethoprim were purified and then transformed with the pMG02 plasmid to exchange the Tn5-B13S by the trimethoprim cassette as described (Marchetti et al. 2010). Trimethoprim-resistant and tetracycline-sensitive transformants were verified by PCR using the oCBM196 and oCBM198 primers.

\section{Plant assays.}

Two lots of $M$. pudica seed collected in the field was obtained from B\&T World Seed (Paguignan, France), the first lot purchased from Thailand and the second from Australia, because seed from Thailand were not available at that time. Thailand seed were used for experimental evolution and coinoculation experiments with two different clones and Australian seed for all other experiments.

Unless stated otherwise, seedlings of $M$. pudica were grown in Gibson tubes under $\mathrm{N}$-free conditions, as previously described (Marchetti et al. 2010), except that tubes contained Fahraeus slant agar and liquid Jensen 1/4.

For competition for nodulation and relative fitness experiments, different combinations of spectinomycin- or kanamycin- 
resistant derivatives of ancestors and final evolved clones were used. Tubes containing two plants were co-inoculated with $10^{4}: 10^{4}$ cells for Thailand seed or $10^{5}: 10^{5}: 10^{5}$ cells for Australian seed. For relative fitness analysis, all surface-sterilized nodules were pooled, crushed, and plated on selective medium. For competition for nodulation analysis, surface-sterilized nodules were individually crushed and plated on selective medium. Number of bacteria recovered from nodules was normalized by the inoculum ratio.

To count the number of viable bacteria per nodule (for fitness measurement), all nodules were harvested at $21 \mathrm{dpi}$, surface sterilized, and crushed, and dilutions were plated on BG medium to recover bacteria, as previously described (Marchetti et al. 2010).

For cytology, nodules were harvested at $21 \mathrm{dpi}$ and cut into $60-\mu \mathrm{m}$ sections with a Leica VT1000S vibratome apparatus. For each nodule, the largest longitudinal section was observed using an inverted microscope (DMIRBE; Leica, Ryswyck, The Netherlands) and images acquired using a CCD camera (Color Coolview, Photonic Science, Milham, U.K.). For semiquantitative infection analyses, sections of nodules induced by ancestral and evolved clones bearing a gusA fusion or a lacZ fusion for $C$ taiwanensis were stained by X-gluc or X-gal respectively. On each section the total nodule surface was measured and the colonized area was quantified by measuring the blue area using the Image-Pro Plus software (Media Cyberbnetics, Rockville, MD, U.S.A.). Results were obtained from 25 to 39 nodules and two independent experiments.

For necrosis analysis, the necrotic zone was identified as a dark-brown zone distinguished from the rest of the nodule. We used 30 to 52 nodules from three different experiments for the CBM212 lineages and two different experiments for the wildtype and nifH $C$. taiwanensis mutant and the CBM349 lineages. Presence of necrotic areas on nodule sections was estimated by visual and expert observation. Quantification of necrosis surfaces was performed by image analysis according to the HSI color model and using Image Pro-Plus software (Media Cyberbnetics).

For persistence analysis, seedlings of $M$. pudica were allowed to grow in $12-\mathrm{cm}^{2}$ plates (three plants per plate) containing slanting nitrogen-free Fahraeus agar medium for 3 days at $28^{\circ} \mathrm{C}$. Roots were covered with a sterile, gas-permeable, and transparent plastic film (BioFolie 25; Sartorius AG, Vivascience, Bedminster, NJ, U.S.A.). Inoculation with bacteria was performed by pipetting an aqueous suspension of exponentially growing bacteria (approximately $10^{3}$ bacteria/ml) between the plastic foil and the semisolid medium.

Nodules were harvested at different time points and embedded in $2 \%(\mathrm{wt} / \mathrm{vol})$ agarose. Nodule sections of $60 \mu \mathrm{m}$ were prepared with a Leica VT1200S vibratome and incubated for $15 \mathrm{~min}$ in live/dead staining solution $(5 \mathrm{mM}$ SYTO9 and 30 $\mathrm{mM}$ PI in $50 \mathrm{mM}$ Tris [pH 7.0] buffer) (Live/Dead BacLight; Invitrogen, Carlsbad, CA, U.S.A.). Sections were removed from the staining solution and mounted in deionized water for microscopy observation. Images were acquired with a fluorescent inverted Leica microscope. Analyses are the results of two independent experiments for a total of 7 to 15 nodules for each time point.

\section{Protection assays of Arabidopsis plants.}

Four-week-old $A$. thaliana susceptible Col-0 plants were root inoculated by the avirulent $h r p G$ mutant of $R$. solanacearum GMI1000 (CBM205) or an evolved clone grown overnight in Phi medium and resuspended in water at a an optical density at $600 \mathrm{~nm}\left(\mathrm{OD}_{600}\right)$ of 0.5 , as described (Deslandes et al. 1998). At $24 \mathrm{~h}$ after the first inoculation, plants were challenged by the virulent GMI1000 strain at $\mathrm{OD}_{600}=0.2$ (approxi- mately $10^{8} \mathrm{CFU} / \mathrm{ml}$ ) and wilting symptoms were monitored for 3 to 4 weeks. Plants were considered dead when more than $50 \%$ of the leaves were wilted. Comparison of disease development was as described previously (Remigi et al. 2011).

\section{ROS analysis.}

In vivo determination of ROS release was performed using H2DCFDA (Molecular Probes, Leiden, The Netherlands), which was dissolved in dimethyl sulfoxide to produce $100 \mathrm{mM}$ stock. Nodules slides $(60 \mu \mathrm{m})$ were placed into 24 -well plates containing $1 \mathrm{ml}$ of loading buffer (Tris $\mathrm{KCl}$ at 10 and $50 \mathrm{mM}$, respectively, $\mathrm{pH} 7.2$ ) and $0.5 \mathrm{ml}$ of $\mathrm{H} 2 \mathrm{DCFDA}$ stock solution and maintained in the dark for $20 \mathrm{~min}$ to obtain basal levels of ROS. Nodules slides were then removed and placed in fresh loading buffer to wash off excess dye. Examination was performed in bright field (or fluorescence) using a Leica MZ FLIII fluorescence stereomicroscope (Leica, Rueil Malmaison, France) (excitation filter $470 / 40 \mathrm{~nm}$, barrier filter $515 \mathrm{~nm}$ ). It should be noted that H2DCFDA exhibits selectivity for $\mathrm{H}_{2} \mathrm{O}_{2}$ over free radicals (Allan and Fluhr 1997); nevertheless, this assay provides an integral measurement for several ROS because it is likely that, in vivo, other radical species are quickly converted to the more stable $\mathrm{H}_{2} \mathrm{O}_{2}$ (Rodriguez-Lopez et al. 2001).

\section{Leghemoglobin analysis.}

For each strain, 20 nodules were ground to fine powder under liquid nitrogen and proteins were extracted with modified Laemmli's sodium dodecyl sulfate (SDS) sample buffer $(10 \%$ SDS, 0.5 M Tris-Cl [pH 6.8], 10\% glycerol, $1 \%$ bromophenol blue, and 20\% 2-mercaptoethanol). Protein concentrations were determined by the BRADFORD method (Bensadoun and Weinstein 1976). Proteins $(20 \mu \mathrm{g})$ were subjected to SDS polyacrylamide gel electrophoresis on a $12 \%$ (wt/vol) polyacrylamide gel and were electroblotted onto an Immobilon-P membrane (Millipore, Bedford, MA, U.S.A.), according to the manufacturer's instructions. Membranes were reacted with antisera raised against leghemoglobin $\mathrm{C}$ of soybean followed by detection by anti-rabbit immunoglobulin G (Sigma-Aldrich, St. Louis) conjugated with HRP and revealed by chemioluminescent system (Roche, Indianapolis, IN, U.S.A.).

\section{Statistical analyses.}

Statistical analyses for infection, necrosis, and fitness were performed using the $\mathrm{R}$ software and the nonparametric statistical test of Kruskal-Wallis, with a value threshold of 0.05 .

\section{ACKNOWLEDGMENTS}

We thank G. Sarath for providing leghemoglobin antibodies and C.-C. Tseng for constructing strain CBM2432. This work was supported by funds from ANR-12-ADAP-0014-01 and the French Laboratory of Excellence project "TULIP" (ANR-10-LABX-41 and ANR-11-IDEX-0002-02).

\section{LITERATURE CITED}

Allan, A. C., and Fluhr, R. 1997. Two distinct sources of elicited reactive oxygen species in tobacco epidermal cells. Plant Cell 9:15591572 .

Amadou, C., Pascal, G., Mangenot, S., Glew, M., Bontemps, C., Capela, D., Carrere, S., Cruveiller, S., Dossat, C., Lajus, A., Marchetti, M., Poinsot, V., Rouy, Z., Servin, B., Saad, M., Schenowitz, C., Barbe, V., Batut, J., Medigue, C., and Masson-Boivin, C. 2008. Genome sequence of the beta-rhizobium Cupriavidus taiwanensis and comparative genomics of rhizobia. Genome Res. 18:1472-1483.

Barrick, J. E., and Lenski, R. E. 2013. Genome dynamics during experimental evolution. Nat. Rev. Genet. 14:827-839.

Barrick, J. E., Yu, D. S., Yoon, S. H., Jeong, H., Oh, T. K., Schneider, D., Lenski, R. E., and Kim, J. F. 2009. Genome evolution and adaptation 
in a long-term experiment with Escherichia coli. Nature 461:12431274.

Bensadoun, A., and Weinstein, D. 1976. Assay of protein in presence of interfering materials. Anal. Biochem. 70:241-250.

Berendsen, R. L., Pieterse, C. M. J., and Bakker, P. 2012. The rhizosphere microbiome and plant health. Trends Plant Sci. 17:478-486.

Boucher, C. A., Barberis, P. A., Trigalet, A. P., and Demery, D. A. 1985. Transposon mutagenesis of Pseudomonas solanacearum-Isolation of TN5-induced avirulent mutants. J. Gen. Microbiol. 131:2449-2457.

Buckling, A., Maclean, R. C., Brockhurst, M. A., and Colegrave, N. 2009. The Beagle in a bottle. Nature 457:824-829.

Bulgarelli, D., Rott, M., Schlaeppi, K., van Themaat, E. V. L., Ahmadinejad, N., Assenza, F., Rauf, P., Huettel, B., Reinhardt, R., Schmelzer, E. Peplies, J., Gloeckner, F. O., Amann, R., Eickhorst, T., and SchulzeLefert, P. 2012. Revealing structure and assembly cues for Arabidopsis root-inhabiting bacterial microbiota. Nature 488:91-95

Capela C., Guan S., and Masson-Boivin C. 2013. In: Diversity and Evolution of Nitrogen-Fixing Legume Symbionts. F. de Bruijn, ed. WileyBlackwell, Hoboken, NJ, U.S.A.

Chen, W. M., Moulin, L., Bontemps, C., Vandamme, P., Bena, G., and Boivin-Masson, C. 2003. Legume symbiotic nitrogen fixation by beta-proteobacteria is widespread in nature. J. Bacteriol. 185:72667272 .

Deslandes, L., Pileur, F., Liaubet, L., Camut, S., Can, C., Williams, K., Holub, E., Beynon, J., Arlat, M., and Marco, Y. 1998. Genetic characterization of RRS1, a recessive locus in Arabidopsis thaliana that confers resistance to the bacterial soilborne pathogen Ralstonia solanacearum. Mol. Plant-Microbe Interact. 11:659-667.

Dixon, R., and Kahn, D. 2004. Genetic regulation of biological nitrogen fixation. Nat. Rev. Microbiol. 2:621-631.

Elliott, G. N., Chou, J. H., Chen, W. M., Bloemberg, G. V., Bontemps, C., Martinez-Romero, E., Velazquez, E., Young, J. P. W., Sprent, J. I., and James, E. K. 2009. Burkholderia spp. are the most competitive symbionts of Mimosa, particularly under N-limited conditions. Environ. Microbiol. 11:762-778.

Felix, G., Duran, J. D., Volko, S., and Boller, T. 1999. Plants have a sensitive perception system for the most conserved domain of bacterial flagellin. Plant J. 18:265-276.

Feng, D. X., Tasset, C., Hanemian, M., Barlet, X., Hu, J., Tremousaygue, D., Deslandes, L., and Marco, Y. 2012. Biological control of bacterial wilt in Arabidopsis thaliana involves abscissic acid signalling. New Phytol. 194:1035-1045.

Fones, H., and Preston, G. M. 2012. Reactive oxygen and oxidative stress tolerance in plant pathogenic Pseudomonas. FEMS (Fed. Eur. Microbiol. Soc.) Microbiol. Lett. 327:1-8.

Galibert, F., Finan, T. M., Long, S. R., Puhler, A., Abola, P., Ampe, F., Barloy-Hubler, F., Barnett, M. J., Becker, A., Boistard, P., Bothe, G., Boutry, M., Bowser, L., Buhrmester, J., Cadieu, E., Capela, D., Chain, P., Cowie, A., Davis, R. W., Dreano, S., Federspiel, N. A., Fisher, R. F., Gloux, S., Godrie, T., Goffeau, A., Golding, B., Gouzy, J., Gurjal, M., Hernandez-Lucas, I., Hong, A., Huizar, L., Hyman, R. W., Jones, T., Kahn, D., Kahn, M. L., Kalman, S., Keating, D. H., Kiss, E., Komp, C. Lalaure, V., Masuy, D., Palm, C., Peck, M. C., Pohl, T. M., Portetelle, D., Purnelle, B., Ramsperger, U., Surzycki, R., Thebault, P., Vandenbol, M., Vorholter, F. J., Weidner, S., Wells, D. H., Wong, K., Yeh, K. C., and Batut, J. 2001. The composite genome of the legume symbiont Sinorhizobium meliloti. Science 293:668-672.

Gehlot, H. S., Tak, N., Kaushik, M., Mitra, S., Chen, W. M., Poweleit, N., Panwar, D., Poonar, N., Parihar, R., Tak, A., Sankhla, I. S., Ojha, A., Rao, S. R., Simon, M. F., Reis Junior, F. B., Perigolo, N., Tripathi, A. K., Sprent, J. I., Young, J. P. W., James, E. K., and Gyaneshwar, P. 2013. An invasive Mimosa in India does not adopt the symbiont of its native relatives. Ann. Bot. 112:179-96.

Gibson, K. E., Kobayashi, H., and Walker, G. C. 2008. Molecular determinants of a symbiotic chronic infection. Annu. Rev. Genet. 42:413441

Guan, S. H., Gris, C., Cruveiller, S., Pouzet, C., Tasse, L., Leru, A., Maillard, A., Medigue, C., Batut, J., Masson-Boivin, C., and Capela, D. 2013. Experimental evolution of nodule intracellular infection in legume symbionts. ISME J. 7:1367-1377.

Gyaneshwar, P., Hirsch, A. M., Moulin, L., Chen, W. M., Elliott, G. N., Bontemps, C., Estrada-de los Santos, P., Gross, E., dos Reis, F. B. Sprent, J. I., Young, J. P. W., and James, E. K. 2011. Legume-nodulating betaproteobacteria: Diversity, host range, and future prospects. Mol. Plant-Microbe Interact. 24:1276-1288.

Hirsch, A., Lum, M., and Downie, J. 2001. What makes the rhizobia-legume symbiosis so special? Plant Physiol. 127:1484-1492.

Ivanov, S., Fedorova, E., and Bisseling, T. 2010. Intracellular plant microbe associations: Secretory pathways and the formation of perimicrobial compartments. Curr. Opin. Plant Biol. 13:372-377.
Jones, J. D. G., and Dangl, J. L. 2006. The plant immune system. Nature 444:323-329.

Jones, K. M., Kobayashi, H., Davies, B. W., Taga, M. E., and Walker, G.C. 2007. How rhizobial symbionts invade plants: The SinorhizobiumMedicago model. Nat. Rev. Microbiol. 5:619-633.

Kiers, E., Rousseau, R., West, S., and Denison, R. 2003. Host sanctions and the legume-rhizobium mutualism. Nature 425:78-81.

Lamb, C., and Dixon, R. A. 1997. The oxidative burst in plant disease resistance. Annu. Rev. Plant Physiol. Plant Mol. Biol. 48:251-275.

Le Gac, M., Cooper, T. F., Cruveiller, S., Edigue, C. M., and Schneider, D. 2013. Evolutionary history and genetic parallelism affect correlated responses to evolution. Mol. Ecol. 22:3292-3303.

Lenski, R. E., and Travisano, M. 1994. Dynamics of adaptation and diversification-A 10,000 generation experiment with bacterial populations. Proc. Natl. Acad. Sci. U.S.A. 91:6808-6814.

Lundberg, D. S., Lebeis, S. L., Paredes, S. H., Yourstone, S., Gehring, J. Malfatti, S., Tremblay, J., Engelbrektson, A., Kunin, V., del Rio, T. G., Edgar, R. C., Eickhorst, T., Ley, R. E., Hugenholtz, P., Tringe, S. G., and Dangl, J. L. 2012. Defining the core Arabidopsis thaliana root microbiome. Nature 488:86-90.

Marchetti, M., Capela, D., Glew, M., Cruveiller, S., Chane-Woon-Ming, B., Gris, C., Timmers, T., Poinsot, V., Gilbert, L. B., Heeb, P., Medigue, C., Batut, J., and Masson-Boivin, C. 2010. Experimental evolution of a plant pathogen into a legume symbiont. PLoS Biol. 8:e1000280

Marchetti, M., Catrice, O., Batut, J., and Masson-Boivin, C. 2011. Cupriavidus taiwanensis bacteroids in Mimosa pudica indeterminate nodules are not terminally differentiated. Appl. Environ. Microbiol. 77:21612164

Masson-Boivin, C., Giraud, E., Perret, X., and Batut, J. 2009. Establishing nitrogen-fixing symbiosis with legumes: How many rhizobium recipes? Trends Microbiol. 17:458-466.

Mithofer, A. 2002. Suppression of plant defence in rhizobia-legume symbiosis. Trends Plant Sci. 7:440-444.

Moreau, S., Verdenaud, M., Ott, T., Letort, S., de Billy, F., Niebel, A., Gouzy, J., de Carvalho-Niebel, F., and Gamas, P. 2011. Transcription reprogramming during root nodule development in Medicago truncatula. PLoS One 6:e16463.

Moulin, L., Munive, A., Dreyfus, B., and Boivin-Masson, C. 2001. Nodulation of legumes by members of the beta-subclass of Proteobacteria. Nature 411:948-950.

Oke, V., and Long, S. R. 1999. Bacteroid formation in the RhizobiumLegume symbiosis. Curr. Opin. Microbiol. 2:641-646.

Oldroyd, G. E. D., and Downie, J. M. 2008. Coordinating nodule morphogenesis with rhizobial infection in legumes. Annu. Rev. Plant Biol. 59:519-546.

Oono, R., Anderson, C. G., and Denison, R. F. 2011. Failure to fix nitrogen by non-reproductive symbiotic rhizobia triggers host sanctions that reduce fitness of their reproductive clonemates. Proc. Royal Soc. B Biol. Sci. 278:2698-2703.

Ramsay, J. P., Sullivan, J. T., Jambari, N., Ortori, C. A., Heeb, S., Williams, P., Barrett, D. A., Lamont, I. L., and Ronson, C. W. 2009. A LuxRI-family regulatory system controls excision and transfer of the Mesorhizobium loti strain R7A symbiosis island by activating expression of two conserved hypothetical genes. Mol. Microbiol. 73:11411155

Remigi, P., Anisimova, M., Guidot, A., Genin, S., and Peeters, N. 2011. Functional diversification of the GALA type III effector family contributes to Ralstonia solanacearum adaptation on different plant hosts New Phytol. 192:976-987.

Rodriguez-Lopez, J. N., Lowe, D. J., Hernandez-Ruiz, J., Hiner, A. N. P., Garcia-Canovas, F., and Thorneley, R. N. F. 2001. Mechanism of reaction of hydrogen peroxide with horseradish peroxidase: Identification of intermediates in the catalytic cycle. J. Am. Chem. Soc. 123:1183811847.

Rogel, M., Hernández-Lucas, I., Kuykendall, L., Balkwill, D., and MartinezRomero, E. 2001. Nitrogen-fixing nodules with Ensifer adhaerens harboring Rhizobium tropici symbiotic plasmids. Appl. Environ. Microbiol. 67:3264-3268.

Saeki, K. 2011. Rhizobial measures to evade host defense strategies and endogenous threats to persistent symbiotic nitrogen fixation: A focus on two legume-rhizobium model systems. Cell. Mol. Life Sci. 68:13271339.

Sprent, J. I. 2001. Nodulation in Legumes. Royal Botanic Gardens, Kew, U.K.

Sprent, J. I. 2007. Evolving ideas of legume evolution and diversity: A taxonomic perspective on the occurrence of nodulation. New Phytol. $174: 11-25$

Sullivan, J., and Ronson, C. 1998. Evolution of rhizobia by acquisition of a 500-kb symbiosis island that integrates into a phe-tRNA gene. Proc. 
Natl. Acad. Sci. U.S.A. 95:5145-5149.

Sy, A., Giraud, E., Jourand, P., Garcia, N., Willems, A., de Lajudie, P., Prin, Y., Neyra, M., Gillis, M., Boivin-Masson, C., and Dreyfus, B. 2001. Methylotrophic Methylobacterium bacteria nodulate and fix nitrogen in symbiosis with legumes. J. Bacteriol. 183:214-220.

Terpolilli, J. J., Hood, G. A., and Poole, P. S. 2012. What determines the efficiency of $\mathrm{N}_{2}$-fixing rhizobium-legume symbioses? Adv. Microb. Physiol. 60:325-389.

Tian, C. F., Zhou, Y. J., Zhang, Y. M., Li, Q. Q., Zhang, Y. Z., Li, D. F., Wang, S., Wang, J., Gilbert, L. B., Li, Y. R., and Chen, W. X. 2012. Comparative genomics of rhizobia nodulating soybean suggests extensive recruitment of lineage-specific genes in adaptations. Proc. Natl.
Acad. Sci. U.S.A. 109:8629-8634

Timmers, A. C. J., Soupene, E., Auriac, M. C., de Billy, F., Vasse, J., Boistard, P., and Truchet, G. 2000. Saprophytic intracellular rhizobia in alfalfa nodules. Mol. Plant-Microbe Interact. 13:1204-1213.

Udvardi, M., and Poole, P. S. 2013. Transport and metabolism in legumerhizobia symbioses. Annu. Rev. Plant Biol. 64:781-805.

Virta, M., Lineri, S., Kankaapaa, P., Karp, M., Peltonen, K., Nuutila, J., and Lilius, E. M. 1998. Determination of complement-mediated killing of bacteria by viability staining and bioluminescence. Appl. Environ. Microbiol. 64:515-519.

Zamioudis, C., and Pieterse, C. M. J. 2012. Modulation of host immunity by beneficial microbes. Mol. Plant-Microbe Interact. 25:139-150. 